\title{
Biomarkers, Epigenetics and Pancreatic Cancer
}

\section{Marco Falasca*}

Queen Mary University of London, Barts and The London School of Medicine and Dentistry, Blizard Institute, Inositide Signalling Group, London E1 2AT, UK

Epigenetics, as brilliantly described by the biologist Nessa Carey in her recent book, is the new discipline that is revolutionising biology and, inevitably, the biomarker field. Epigenetics can be described as changes in the genome that do not involve modification of DNA sequence. Typical epigenetic modifications are the methylation of DNA and modification of histones, proteins closely associated with DNA. A mutual regulation links epigenetic modifications to micro RNAs (miRNAs). The miRNAs are small, around 19-25 nucleotides long, non coding RNAs that regulate gene expression post-trascriptionally. Recent studies have demonstrated that DNA methylation and histone modifications regulate miRNAs. On the other hand miRNAs control the expression of key epigenetic regulators such as DNA methyltransferases and histone deacetylases. Therefore, this epigenetic-miRNA connection forms a complex regulatory circuit that regulates the whole gene expression profile. Interestingly, miRNAs are not only present inside cells but also outside cells including body fluids. Furthermore, miRNAs are surprisingly stable, given the instability of most RNA molecules, and possess several other requisites for a good biomarker molecule such as tissue specificity and methods of assessment (PCR). Last but not least, changes of miRNA levels in body fluids such as plasma, serum, saliva and urine, have been associated with several diseases including cancer. Among the different cancer types, the potential diagnostic utility of miRNAs has attracted particular attention in the pancreatic cancer (PC) field. PC has one of the poorest prognoses among all cancers partly because of its silent nature and tendency for late discovery. Therefore, identification of biomarkers associated with the early stage of the disease is critical in order to detect the malignancy promptly. Despite the fact that several biomarkers having been proposed for PC, the serum carbohydrate antigen CA $19-9$ is the only available biomarker in the clinic with around $90 \%$ specificity and $81 \%$ sensitivity to PC. Nevertheless, the use of CA 19-9 for PC prognosis and patients' monitoring is disputed. Therefore, novel biomarkers with higher specificity for PC are required. In recent years, several studies have assessed the miRNA profile in PC tumour tissue, compared to chronic pancreatitis and normal pancreas. These studies have underlined the great potential of miRNA as biomarker in PC. On the other hand, some issues remain to be solved in order to propose miRNA as a clinical biomarker. Over a thousand miRNAs have been identified in human tissues and not surprisingly, the miRNA profiles emerged from different studies in PC show a differential expression and non overlapping results. This may be due to the fact that pancreatic tissues are highly homogenous and therefore difference in tissue sampling for instance, may impact the result. Consequently, sample standardization is required in order to develop a more accurate assessment of miRNA quality and quantity in PC specimens. Further studies are required to define a clear and reproducible miRNA pattern that will help to predict patient survival. The potential of the diagnostic value of miRNA, particularly in PC, is very attractive and new perspectives are still to be fully explored. For instance, the prospect to study miRNA in the so-called circulating tumour cells or in the cancer stem cells is particularly attractive.
*Corresponding author: Marco Falasca, Queen Mary University of London, Barts and The London School of Medicine and Dentistry, Blizard Institute, Inositide Signalling Group, London E1 2AT, UK, E-mail: m.falasca@qmul.ac.uk

Received September 25, 2012; Accepted September 26, 2012; Published September 28, 2012

Citation: Falasca M (2012) Biomarkers, Epigenetics and Pancreatic Cancer. J Mol Biomark Diagn 3:e113. doi:10.4172/2155-9929.1000e113

Copyright: () 2012 Falasca M. This is an open-access article distributed under the terms of the Creative Commons Attribution License, which permits unrestricted use, distribution, and reproduction in any medium, provided the original author and source are credited 